\title{
Limitaciones y desafíos del quehacer de las y los cientistas sociales en el capitalismo informacional: Un abordaje desde el análisis de tres experiencias de investigación
}

\author{
Agostina Dolcemascolo \\ Consejo Nacional de Investigaciones Científicas y Técnicas, Argentina \\ agostinadolcemascolo@gmail.com \\ LUCILA DUGHERA \\ Consejo Nacional de Investigaciones Científicas y Técnicas, Argentina \\ ludughera@gmail.com
}

DOI 10.11606/issn.2316-9133.v29i2pe175285

resumen El objetivo de este trabajo es recuperar y analizar tres experiencias de investigación para pensar los obstáculos, problemas y desafíos a los que nos enfrentamos como cientistas sociales al abordar fenómenos vinculados a las tecnologías digitales e internet. En este sentido, proponemos una reflexión situada que ponga en diálogo las características de las tecnologías digitales e internet con algunos aspectos de nuestra experiencia como investigadoras. A partir de este objetivo, hacemos foco en tres aspectos de nuestro quehacer: cómo recortar el objeto, cómo acceder a los datos y cómo recolectar la información. Adicionalmente, nos ocupamos de abordar un aspecto transversal a los anteriores que refiere a la cuestión ética para preguntarnos sobre cuál es el límite de aquello que observamos y analizamos en este tipo de investigaciones.

palabras clave Tecnologías digitales. Internet. Metodología de investigación. Experiencias. Ciencias sociales.

Limitations and challenges of social scientists' work in informational capitalism: an approach regarding three research experiences

abstract The aim of this paper is to recover and analyze three research experiences to think about the obstacles, problems and challenges that we face as social scientists when dealing with phenomena related to digital technologies and the internet. In this sense, we propose a situated reflection that puts the characteristics of digital technologies and the internet in dialogue with some aspects of our experience as researchers. To do this, we focus on three aspects: how to define the object, how to access the data, and 
how to collect the information. Additionally, we deal with addressing a cross-cutting aspect that refers to the ethical question and places the question about what is the limit of what we observe and analyze in this type of research.

keywords Digital technologies. Internet. Research methodology. Experiences. Social Sciences

\section{Limitações e desafios do trabalho dos cientistas sociais no capitalismo} informacional: uma abordagem a partir da análise de três experiências de pesquisa

resumo $\mathrm{O}$ objetivo deste trabalho é resgatar e analisar três experiências de pesquisa para pensar os obstáculos, problemas e desafios que enfrentamos como cientistas sociais ao lidar com fenômenos relacionados às tecnologias digitais e à internet. Nesse sentido, propomos uma reflexão situada que coloca as características das tecnologias digitais e da internet em diálogo com alguns aspectos de nossa experiência como pesquisadores. Para fazer isso, nos concentramos em três aspectos: como delimitar o objeto, como acessar os dados e como coletar as informações. Além disso, tratamos de abordar um aspecto transversal aos anteriores que remete à questão ética de nos perguntarmos qual é o limite do que observamos e analisamos neste tipo de pesquisa.

palavras-chaves Tecnologias digitais. Internet. Metodologia de pesquisa. Experiências. Ciências Sociais.

\section{Introducción}

"Si las teorías, los conceptos y los métodos no se renuevan, si no incorporan nuevos temas, preguntas y búsqueda de respuestas, pierden interés" (SAUTÚ 2011: p.60)

'Las tecnologías digitales ${ }^{1}$ e internet han traído aparejado una diversidad de transformaciones, tensiones en las distintas dimensiones de la vida social, económica, política y subjetiva. La producción de conocimiento en el campo de las ciencias sociales ${ }^{2}$ no resulta ajena a estas. En efecto, es posible identificar por lo menos dos grandes discusiones

\footnotetext{
${ }^{1}$ Las tecnologías digitales "son aquellas que procesan, transmiten, almacenan o generan información digital (ID). La definimos como toda forma de conocimiento codificado binariamente mediante señales eléctricas de encendido-apagado" (ZUKERFELD 2007: p.41).

${ }^{2}$ En esta oportunidad, dado el alcance del trabajo y el tipo de propuesta que realizamos, nos abocamos a las ciencias sociales únicamente. Aunque, entendemos que en las ciencias humanas algunos de los aspectos aquí propuestos también acontecen.
} 
al respecto. Claro que cada una de ellas con diferentes niveles de incidencia y alcance. La primera reside en cuáles son las estrategias metodológicas, junto con los instrumentos de recolección de datos, que nos permiten dar cuenta de nuestros objetos de investigación de manera "más acabada". La segunda consiste en identificar qué aspectos y en qué medida las teorías sociales y humanas siguen resultándonos fructíferas para analizar las diferentes dimensiones de la vida social que se van co-construyendo a partir de, y junto con, las tecnologías digitales e internet. En este sentido, Saskia Sassen (2002, p.365) señala que "lidiar con la tecnología digital implica también la tarea de desarrollar las categorías analíticas que permitan capturar las imbricaciones complejas entre tecnología y sociedad."

Si bien ambas discusiones nos resultan altamente estimulantes y se hallan imbricadas, en esta ocasión nos dedicamos a la primera. Más aún, las líneas que siguen articulan las características de las tecnologías digitales e internet con algunos aspectos de nuestra experiencia como investigadoras que, en ocasiones, no solo han tensionado dicho transitar, sino que la mayoría de las veces quedan opacados. Por ejemplo, las dificultades que enfrentamos para recortar nuestros objetos de estudio, recolectar datos, construir instrumentos que sean capaces de relevar la gran variedad de datos que ofrece cada fenómeno, entre otras. Así, se ponen en común y, a la vez, se problematizan algunos de estos aspectos desde el espacio autobiográfico ${ }^{3}$. Es decir, "se trata aquí de pensar la experiencia y desde la experiencia [...] La experiencia supone un acontecimiento exterior a mí. Pero el lugar de la experiencia es en mí" (LARROSA 2006: p.87-89). En efecto, este trabajo propone realizar una reflexión situada acerca de los métodos e instrumentos de recolección de datos utilizados para estudiar lo que sucede en internet y con las tecnologías digitales a partir de reponer algunas de las investigaciones en las que hemos participado, junto con sus respectivos instrumentos de recolección de datos. Específicamente, ponemos el foco en tres aspectos particulares: cómo recortar el objeto, cómo acceder a los datos y cómo recolectar la información. Adicionalmente, nos ocupamos de abordar un aspecto transversal a los anteriores que refiere a la cuestión ética para preguntarnos sobre cuál es el límite de aquello que observamos y analizamos en este tipo de investigaciones.

Las preguntas que vertebran este escrito son: ¿qué diálogos es posible establecer entre las características intrínsecas de las tecnologías digitales y la producción de conocimiento social?, ¿cuál es la particularidad de las investigaciones que abordan y problematizan las tecnologías digitales en todas sus dimensiones?, ¿cuáles son las nuevas metodologías disponibles que parecerían facilitar la producción de conocimiento social actualmente?, ¿en qué sentidos?, ¿cómo hemos sorteado las dificultades propias de este tipo de objetos, como puede ser, la falta de presencialidad en algunos casos, la velocidad con la que cambian

\footnotetext{
${ }^{3}$ Entendemos este espacio, "(n)o solo como referente de identidad o como forma privilegiada de expresión de la singularidad, sino también y principalmente como aquello que nos permite reconstruir acontecimientos del pasado y dotar de un sentido nuevo la relación con los otros, nuestra propia existencia" (RUIZ SILVA 2016: pp. 5-6).
} 
plataformas, los usos, etc., entre otras cuestiones?, ¿qué desafíos éticos nos implica la producción de conocimiento social en el capitalismo informacional?

En resumen, el artículo se estructura de la siguiente manera. Primero, definimos qué entendemos por tecnologías digitales (TD) y cuáles son sus especificidades. En este mismo apartado, presentamos algunas innovaciones metodológicas en el campo de las ciencias sociales vinculadas al estudio de y a través de las TD. Segundo, describimos tres experiencias de investigación en las que participamos para analizar, seguido de esto, las dificultades con las que nos enfrentamos a nivel metodológico a partir de los aspectos antes especificados. Cerramos este escrito con algunas propuestas, así como nuevos interrogantes, para pensar y explorar formas de abordar lo metodológico que permitan sortear este tipo de obstáculos.

\section{Tecnologías digitales: algunas definiciones y métodos innovadores}

En las últimas décadas, los discursos acerca de las tecnologías digitales e internet ligados a la problematización de las formas de producción de conocimiento y con ella a los bienes que éstas producen, bienes informacionales ${ }^{4}$, han cobrado relevancia tanto en la escena académica regional como internacional. Estas transformaciones cristalizan en una nueva forma de organización social en la que la generación, el procesamiento y la transmisión de la información se convierten en las fuentes fundamentales de la productividad y el poder (VILLAR ONRUBIA 2005). Dichas transformaciones se enmarcan en una nueva etapa del modo de producción capitalista, entendida aquí como informacional. Específicamente, en esta "la fuente de productividad estriba en la tecnología de la generación de conocimiento, el procesamiento de la información y la comunicación de símbolos" (CASTELLS 2008: p.43). Es decir, lo particular de esta etapa del modo de producción capitalista, entendida aquí como informacional, reside en que dicha información se objetiva en bienes hechos de pura información digital y soportes digitales (CASTELLS 2008 [1997]; ZUKERFELD 2010).

Claro que hasta aquí parecería ser simplemente un cambio de soportes en los que se objetiva la información. Es decir, pasamos de la preeminencia de soportes analógicos a los digitales, por ejemplo, cambiamos la máquina de escribir por la computadora. No obstante, en este escrito enfatizamos que las características materiales de las tecnologías digitales, junto con las regulaciones ${ }^{5}$ que estas conllevan y los actores que las desarrollan, requieren ser

\footnotetext{
4 Definimos a los BI como "aquellos bienes obtenidos en procesos productivos cuya función de producción está signada por un importante peso relativo de los gastos (en capital o trabajo) en producción y acceso a la ID” (ZUKERFELD 2007). En este artículo nos referimos a un tipo particular de bienes informacionales que llamamos primarios. Estos son bienes informacionales hechos exclusivamente de información digital. Para simplificar la lectura, nos referiremos a ellos aludiéndolos de manera imprecisa como bienes informacionales.

${ }^{5} \mathrm{Si}$ bien en este escrito no nos detenemos sobre las regulaciones que prevalecen sobre la información digital, conviene señalar que, dadas sus características, la propiedad intelectual (PI) pasa a ocupar un lugar preponderante como mecanismo que permite crear valor. Así, en el capitalismo informacional, se identifica un crecimiento cualitativo y cuantitativo de la PI. Más aún, las regulaciones establecidas a través de la PI como
} 
revisitadas ya que podrían permitirnos enriquecer tanto los desafíos y las limitaciones como las posibles "soluciones"6 que hemos ensayado en la producción de conocimiento social.

En este sentido, las TD permiten producir, copiar, almacenar y distribuir conocimiento en soporte digital con un costo cercano a cero. Específicamente, estas tienen como materia prima, y de riqueza, a la información digital (ID). Así, se entiende a la ID como toda aquella forma de conocimiento susceptible de ser digitalizada (VARIAN 1998). Es decir, codificada en términos binarios (CHARTRAND 2005) y con la particularidad de replicarse a un costo cercano a cero (RULLANI 1999; BOUTANG 1999; BLOUNDEU 1999). Asimismo, resultan disruptivas en términos de espacio y tiempo, ya que permiten "romper" con las barreras espacio-temporales, y todo tiempo se torna susceptible de ser productivo. En otras palabras, difuminan la frontera entre el tiempo de trabajo y el tiempo de ocio, o lo que suele denominarse una imbricación entre economía y cultura. Dichas características conviven con dos rasgos particulares de internet: por un lado, su reticularidad potencia la cantidad de información digital disponible y, por otro, su acentrismo dificulta limitar el volumen de ID que circula por la red (VERCELLI 2006).

Otro de los elementos relevantes y disruptivos de las TD e internet, que entendemos tensionan nuestro quehacer como cientistas sociales, es la velocidad con que estas cambian. Específicamente, la tendencia descrita por la Ley de Moore señala que cada 18 meses la capacidad de los procesadores se duplica manteniendo su precio. Es decir, las computadoras, o cualquier otra tecnología digital, se vuelven más potentes y más baratas cada aproximadamente dos años. Esto repercute en la masificación de las TD y en los usos que se van co-construyendo de las mismas (tipo de consumos, interacciones, etc.). A esto se le suman otros cambios marcados por la estabilidad o no de los mercados, el éxito de determinadas aplicaciones, plataformas, restricciones en los usos, etc., que inciden en la más bien breve durabilidad y estabilidad de las TD.

Con todo, a partir del contexto y las características presentadas es posible advertir que las tecnologías digitales e internet pueden jugar un triple papel en la producción de conocimiento social. Específicamente, hallamos hasta el momento tres formas posibles de estar "jugando" allí: a) como instrumento de recolección de datos únicamente; b) como objeto de estudio de alguna dimensión de estas tecnologías, por ejemplo, el uso de redes sociales, las prácticas de enseñanza y aprendizaje mediadas por $\mathrm{TD}$, entre otras; c) como instrumento y objeto al mismo tiempo.

estrategia del capital para restringir el acceso a los conocimientos y volverlos un bien escaso se configuran como lo que algunos autores sentencian bajo el nombre de "cercamientos de la inteligencia colectiva" (RODRÍGUEZ; SÁNCHEZ 2004).

${ }^{6}$ Cabe aclarar que "los problemas y las relaciones de correspondencia problema-solución no son inmanentes a las tecnologías y artefactos sino construcciones sociotécnicas. La relación problema-solución condiciona el conjunto de prácticas sociales e institucionales, en particular: las valorizaciones, las dinámicas de aprendizaje y la generación de formas organizacionales" (SANTOS; THOMAS 2012: p.117). 
Estas especificidades del mundo digital (replicabilidad, perennidad, reticularidad, acentrismo, y velocidad de cambio -Ley de Moore-), junto con la cada vez mayor cantidad de sujetos conectados, la escala de los proyectos colectivos y creativos, desarrollados en (dentro) del universo digital, y a partir de las TD e internet, posibilitan que la producción incesante, gratuita y continua de información digital se potencie y se vuelva clave para el entramado social. Dicho contexto ha traccionado una diversidad de debates y reflexiones en torno al uso de este tipo de tecnologías en la recolección, registro y análisis de los datos. Y esto, dado que tanto la dimensión subjetiva como aquello que entendemos por lo social (valores, lenguaje, normas, redes de reconocimiento, etc.) no solo se hallan generalmente mediados por estas y objetivadas en este tipo de soportes, sino que además van tallando formas de habitar diferentes a las de tiempos pretéritos. Así, es posible imaginar las diferentes tensiones y dificultades con las que lidiamos los y las cientistas sociales al momento de establecer recortes en nuestros objetos de investigación y captar estos fenómenos "vivos", por ejemplo, cuando en el transcurso de nuestra investigación la plataforma que estudiamos cambia sus términos y condiciones, diseño, etc.

Frente a estas dificultades y limitaciones, cabe reponer algunas herramientas metodológicas vinculadas al estudio de TD e internet que han cobrado relevancia en los últimos años en los estudios sociales y que resultan en cierta medida innovadoras. Nos referimos a las etnografias digitales y al uso cada vez más frecuente por parte de los cientistas del big data y la minería de datos. Si bien este artículo no propone discutir estas herramientas, nos interesa dejar aquí una breve referencia que luego será retomada en las conclusiones a la luz del análisis de nuestras experiencias.

Por etnografias digitales entendemos, siguiendo a Di Próspero y Daza (2019), técnicas que incorporan lo digital, las cuales implican

contactarnos con los sujetos de investigación a través de comunicaciones mediadas digitalmente: podemos estar mirando y trackeando digitalmente lo que ellos hacen, podemos preguntar si nos aceptan en sus redes sociales, escuchar puede implicar leer o percibir y comunicar de otras formas, podemos observar y ser observados al mismo tiempo, la toma de notas etnográficas puede ser reemplazada por videos, audios, fotografía o blogs (...). (DI PRÓSPERO; DAZA 2019: p.66)

Dada la penetración de internet y las TD en nuestras vidas, los autores señalan que "hacer etnografías de lo digital es etnografiar el mundo contemporáneo.” (p. 66) En términos generales, podríamos agregar que este tipo de técnicas buscan, por un lado, sortear las limitaciones de las técnicas e instrumentos analógicos y, por otro, aprovechar las características de las TD señaladas anteriormente. En este sentido, no implican un simple traspaso de un medio a otro, sino que se proponen pensar y generar estrategias que dialoguen con los objetos de estudio desde su especificidad digital. 
El big data refiere a la producción, manejo y procesamiento de grandes cantidades de datos. Su definición desde la literatura técnica y mercadológica señala tres atributos técnicos para definirla: volumen (relativa a las grandes cantidades de datos), variedad (distintos tipos de datos) y velocidad (vinculado al continuo flujo en la generación de datos). Esta primera definición, sin embargo, resulta insuficiente para las ciencias sociales. Por un lado, Becerra y López Alurralde (2017), refieren que este es un fenómeno social de suma importancia, y que su progresivo reconocimiento por las ciencias sociales y la consolidación de un subcampo denominado "critical data studies", implica pensar estos datos desde un enfoque crítico, que los contextualice, reconozca su carácter político, tenga en cuenta la compleja relación entre datos y sociedad, las formas en que la academia se acerca a este nuevo régimen de datos y las oportunidades de este acercamiento, entre otras cuestiones. Por otro lado, Menenses Roca señala que existen otras propiedades necesarias para entender el big data, como "la veracidad, objetividad, representatividad y los dilemas éticos asociados.” (2018: p.422)

Aquí también se torna clave la formación necesaria para acceder a este tipo de herramientas. Más aún, las carreras en ciencias sociales en general no nos forman para esto y “...la falta de entrenamiento de los científicos sociales puede traducirse en una claudicación ante las ciencias computacionales en favor de un empirismo que desplace las categorías de pensamiento de las ciencias sociales." (MENENSES ROCA 2018: p.429) Esto tiene además otras consecuencias como es el desarrollo de un mercado privado de lo metodológico, y la producción/recolección de datos sin el manejo de herramientas teóricas para procesarlos, lo que nos lleva a preguntarnos si efectivamente las ciencias sociales pueden devenir en fábrica de producción de datos y, en ese caso, cuál sería su propósito.

En este contexto, nos preguntamos entonces qué otras herramientas aparecen como "disponibles" para los cientistas sociales. Parecería que separando las aguas de los abordajes cualitativos y cuantitativos solo se presentan estas opciones como válidas o, por lo menos, como aquellas que, desde distintas perspectivas y orígenes, ofrecen herramientas para indagar/explorar las TD e internet en el capitalismo informacional. En este sentido, lo que sigue a continuación tiene como propósito reflexionar sobre otras posibles formas de abordar estos temas, o por lo menos, comenzar a pensarlos, deconstruirlos desde otros enfoques que habiliten, eventualmente, el surgimiento de nuevas herramientas metodológicas. Claro que las experiencias que se analizan en ningún caso se igualan a las innovaciones presentadas más arriba, sino que son puestas en común a los fines de comenzar a tejer eventuales desarrollos y de reponer las limitaciones y soluciones que hemos transitado en cada una de ellas.

En resumen, la problematización presentada recupera los rasgos intrínsecos de los soportes digitales para ponerlos a dialogar con características históricas de las metodologías de las ciencias sociales y, al mismo tiempo, reflexiona acerca de las posibles consecuencias de ese desfasaje entre soportes analógicos y digitales. Así, lejos de encontrarnos en una situación estabilizada, se comparten algunas de las inquietudes que nos atraviesan. 


\section{Presentación de los casos: reflexiones a partir de tres experiencias de investigación}

Este apartado tiene como propósito realizar una reflexión situada acerca de las limitaciones y desafíos que hemos experimentado en diferentes investigaciones. En consecuencia, y en pos de ganar claridad expositiva, se divide en dos. En la primera parte, presentamos de manera general dos investigaciones colectivas y una individual en las cuales participamos. Si bien cada una de ellas tiene sus particularidades, comparten que analizan fenómenos sociales que están hechos de información digital y acontecen a partir del uso de soportes digitales. En la segunda parte, sistematizamos los problemas y desafíos que vivenciamos en dichas experiencias de investigación, así como las soluciones propuestas por nosotras. Específicamente, nos circunscribimos a los siguientes ejes ${ }^{7}$ : cómo recortar el objeto, cómo acceder a los datos y cómo recolectar la información. Sumamos a esto, un eje transversal relativo a la cuestión ética.

\section{Primer caso: Investigación A - Flujos de conocimientos, tecnologías digitales y actores sociales en} las escuelas secundarias. Un análisis socio-técnico de las capas del Programa Conectar Igualdad

Este proyecto abordó los impactos en la institución escolar argentina del Programa Conectar Igualdad $(\mathrm{PCI})^{8}$ a partir de tres dimensiones socio-técnicas (subjetiva, intersubjetiva y tecnológica). A nivel metodológico, se combinaron instrumentos cuantitativos y cualitativos: se llevaron a cabo encuestas representativas nacionales a 3183 alumnos y 342 docentes, 8 observaciones no participantes, 32 entrevistas no estructuradas a informantes clave (docentes, directivos y RTE) y 8 focus group (ZUKERFELD; BENITEZ LARGHI 2015).

Respecto a los instrumentos cuantitativos, las encuestas fueron completadas a través de las netbooks del PCI de manera presencial. Es decir, para alcanzar la representatividad del estudio, se coordinó con cada escuela de distintos puntos del país un horario para realizar la encuesta en el curso de la jornada escolar. Esto implicó, además, capacitar a los investigadores en el uso de intranet, dado que la conectividad no estaba asegurada en ninguna institución, y el asesoramiento de un técnico. El cuestionario fue administrado a través del software Limesurvey. En relación al trabajo cualitativo, a partir de las dimensiones mencionadas anteriormente, se observaron clases en las que los y las estudiantes utilizaban las netbooks y

\footnotetext{
${ }^{7}$ Cabe aclarar aquí que dichos ejes en ningún caso agotan la discusión de internet, las tecnologías digitales y la producción de conocimiento social. De cualquier manera, nos resultan aquí de utilidad para dar cuenta tanto de las experiencias transitadas como de las limitaciones y desafíos que hemos transitado.

8 "El PCI consistió no solamente en la entrega de una computadora portátil a estudiantes y docentes de escuelas secundarias de gestión estatal del país -aspecto más visible del programa-, sino además en la provisión de internet, la creación y puesta en disponibilidad de capacitaciones docentes, software y contenidos para la comunidad educativa" (DOLCEMÁSCOLO; DUGHERA; YANSEN 2020: p.44)
} 
se diseñó una guía de focus en la que dichos actores educativos tenían que diseñar una tecnología digital.

\section{Segundo caso: Investigación B - Implicancias y proyecciones de los saberes tecnosociales emergentes en la Educación Superior Argentina}

Esta investigación, aún en curso, tiene como propósito identificar y caracterizar la dimensión cognoscitiva, operativa y aplicativa los saberes tecnosociales (PEIRONE; DUGHERA; BORDIGNON 2019). A nivel metodológico combina instrumentos cualitativos y cuantitativos. En relación a los primeros se realizaron 12 focus group y una actividad denominada "la semana digital". En tanto, en relación a lo cuantitativo, se espera efectuar una encuesta online a estudiantes y docentes.

Los instrumentos cualitativos se diseñaron para captar tanto el nivel discursivo de los saberes tecnosociales como el orden práctico. Con lo cual, fue necesario incorporar en la guía de focus actividades en las que los y las estudiantes habiten problemas y desarrollen, a partir de sus saberes tecnosociales, posibles soluciones. En cuanto a la semana digital, se conformaron grupos de entre 8 y 9 estudiantes cada uno, con dos moderadores que iban dándoles las diferentes actividades a realizar durante los 5 días de trabajo, por ejemplo, grabar tu pantalla, armar una cápsula del tiempo, entre otras. De alguna manera, se intentó emular algunas de las ventajas de la etnografía digital. En consecuencia, para poder participar era necesario contar con conectividad y poseer una tecnología digital con memoria y capacidad de almacenamiento para poder grabar, realizar descargas, instalar algunos programas, etc.

En cuanto a los y las investigadores, algunos requirieron capacitación para la puesta en marcha de la semana digital ya sea en cuestiones técnicas propiamente dichas como en las modalidades de la comunicación con los y las participantes. Asimismo, cabe mencionar que la hechura de esta implicó una diversidad de debates éticos que serán retomados en el apartado que sigue a continuación.

\section{Tercer caso: Investigación $C$ - ¿Trabajar en plataformas de video?: representaciones en torno a la actividad productiva en YouTube}

Esta indagó sobre usos, prácticas, representaciones y discursos en la plataforma de videos Youtube desde la perspectiva de tres de sus actores más representativos: los productores de contenidos audiovisuales, los llamados seguidores y la plataforma de videos. El análisis se realizó en base a cinco dimensiones: trabajo, formación, creatividad, valores y vínculos. Esta investigación, a diferencia de $A$ y $B$, fue de carácter individual y dio lugar a una tesis doctoral (DOLCEMASCOLO 2019).

Metodológicamente, se optó por un abordaje de tipo cuali-cuantitativo, en el cual se contempló la utilización de fuentes primarias y secundarias para cada uno de los actores en intersección con las categorías de análisis propuestas. Para esto, se procedió a: realizar entrevistas semiestructuradas a productores y seguidores de Argentina de manera online (se confeccionó un cuestionario con preguntas abiertas y cerradas en el software Limesurvey y 
se envió a través del correo electrónico); relevar documentos de YouTube Argentina (específicamente, de la sección "Creadores"); efectuar observación no participante de los canales de los productores entrevistados y del sitio; y extraer datos de comunicados de prensa y Estadísticas de YouTube, disponibles en "socialblade.com".

Para la confección de la muestra se partió de utilizar el método denominado "Respondent-driven sampling" (RDS) (HECKATHORN 1997), con el objetivo de poder estimar una "pseudo-población" y establecer un mapa de redes de contactos entre los entrevistados. Este método de muestreo se presenta como una variante del muestreo en cadena o por bola de nieve (MANTECÓN et al. 2008) y se utiliza en los casos de "poblaciones ocultas" ${ }^{\prime \prime}$. De cualquier manera, hallamos que la ausencia de incentivos económicos fue un factor que incidió significativamente para que nuestros entrevistados no nos proveyeran datos de contactos de otros productores/seguidores, y esto resultó en que la aplicación de esta metodología fuera poco viable. Esto nos llevó a recurrir a otras fuentes como la exploración de distintos canales, páginas personales, referencias de conocidos, entre otras, para poder contactar a los posibles entrevistados/as. Así, se realizaron 35 entrevistas a productores (12 mujeres y 19 varones), y 38 de seguidores (21 mujeres y 17 varones).

A partir de las descripciones realizadas, a continuación, nos disponemos a describir las principales problemáticas, obstáculos y desafíos experimentados.

\section{Problemas, obstáculos y desafíos que se presentaron en las investigaciones}

En esta segunda parte, nos interesa reflexionar sobre algunos problemas y obstáculos que hemos enfrentado en nuestras investigaciones vinculadas a las TD e internet. Para ello, tomamos ejemplos de los casos presentados. La búsqueda aquí radica sobre todo en pensar acerca de los desafíos con los que lidiamos como investigadoras al abordar estas temáticas. En la siguiente tabla presentamos la matriz de análisis que utilizamos.

\begin{tabular}{|l|l|l|}
\hline \multirow{2}{*}{$\begin{array}{l}\text { Casos/Ejes de } \\
\text { análisis }\end{array}$} & \multicolumn{2}{|c|}{$(A, B, C)$} \\
\cline { 2 - 3 } & Preguntas iniciales & \multicolumn{2}{c|}{ Aspectos éticos } \\
\hline Recorte del objeto & $\begin{array}{l}\text { ¿Cómo delimitar el objeto ante } \\
\text { tanta información digital } \\
\text { disponible? ¿Qué mirar? }\end{array}$ & $\begin{array}{l}\text { ¿Cuál es el límite de esa supuesta } \\
\text { disponibilidad de información } \\
\text { digicho límite, ¿con qué } \\
\text { está relacionado: cuestiones } \\
\text { técnicas y/o de desconocimiento }\end{array}$ \\
\hline
\end{tabular}

\footnotetext{
${ }^{9}$ Es decir, poblaciones de difícil acceso (por ser estigmatizadas o ilegales) y/o sobre las cuales se desconoce su tamaño (HECKATHORN 1997: p.174). En nuestro caso, suponíamos que la población objetivo no sería reticente a responder o reconocerse como productor o seguidor de YouTube, pero desconocíamos los límites de aquella. Entonces, si bien existe información (aunque estimada) sobre la cantidad de gente que sube y mira videos en YouTube, se desconoce la población que lo hace específicamente en Argentina.
} 


\begin{tabular}{|c|c|c|}
\hline & & y/o éticas, u todas ellas? \\
\hline $\begin{array}{l}\text { Acceso y obtención } \\
\text { de los datos }\end{array}$ & $\begin{array}{l}\text { ¿Cómo accedemos a los datos? } \\
\text { ¿Cuáles son las estrategias para } \\
\text { contactar a los sujetos (por mail, } \\
\text { sitios de redes sociales, etc.)? }\end{array}$ & $\begin{array}{l}\text { ¿Cómo separar al sujeto público } \\
\text { del sujeto privado? ¿Cuáles son } \\
\text { los límites frente a la difuminación } \\
\text { de los espacios públicos/privados? }\end{array}$ \\
\hline $\begin{array}{l}\text { Recolección y } \\
\text { registro de datos }\end{array}$ & $\begin{array}{l}\text { ¿Qué instrumentos resultan } \\
\text { más apropiados para recolectar } \\
\text { datos? ¿Qué limitaciones } \\
\text { presentan en el estudio de } \\
\text { fenómenos vinculados a las TD e } \\
\text { internet? }\end{array}$ & $\begin{array}{l}\text { ¿Hasta dónde es éticamente } \\
\text { válido observar, recolectar, } \\
\text { indagar cuando abordamos el } \\
\text { hacer de sujetos vinculados a las } \\
\text { TD e internet? ¿Cómo sorteamos } \\
\text { dichas tensiones? }\end{array}$ \\
\hline
\end{tabular}

Tabla 1: Matriz de análisis. Fuente: elaboración propia.

A partir de la matriz propuesta, y siguiendo el orden de los ejes, a continuación, analizamos nuestras experiencias a la luz de los ejemplos más representativos de cada investigación $(A, B$ y $C$ ). Este desarrollo, además, dialoga con las características de las TD e internet que abordamos en el apartado anterior, a los fines de precisar la especificidad de este tipo de investigaciones.

En primer lugar, cuando estudiamos fenómenos vinculados a las TD e internet nos enfrentamos a las preguntas de qué mirar y cómo delimitar ese mirar. Por ejemplo, al abordar plataformas como YouTube y/o sitios de redes sociales (Facebook, Instagram, Twitter, etc.) nos encontramos no sólo con la información dispersa, sino también con un volumen imposible de asir de manera manual o artesanal. En efecto, resulta necesario establecer algún tipo de recorte para sistematizar todo ese caudal de datos (sin necesariamente recurrir a herramientas de minería de datos, en el caso de quienes no las manejamos) en función de aquello que nos interesa observar sin caer en la necesidad de reconfigurar nuestro objeto por estas limitaciones. En el caso de la investigación C, las interacciones entre productores y seguidores en YouTube fue abordado solo lateralmente (con algunas pocas ejemplificaciones), dado el volumen de información disponible en el sitio. Quizás, hubiera requerido una investigación aparte que se centrara en indagar en este único aspecto. Cabe señalar aquí que nuestro objetivo principal residía en estudiar las representaciones que estos actores construyen sobre la actividad productiva que tiene lugar en la plataforma. Es por eso, $\mathrm{y}$ en base a las características de las TD e internet, resolvimos descomponerla en cinco dimensiones:

- trabajo: atendimos aquí a la disputa entre quienes consideran las actividades que desarrollan los llamados prosumidores en la web como trabajo y quienes no, destacando su carácter lúdico o de hobby, y las implicancias que uno u otro abordaje tiene. Esto, como mencionamos más arriba, dialoga con la disolución de fronteras entre el tiempo de trabajo y el tiempo de ocio, tan claramente delimitado en el capitalismo industrial; 
- formación: el punto de partida aquí era que muchas actividades vinculadas a la producción de bienes informacionales (producción de software, por ejemplo) se aprenden o perfeccionan en espacios de educación no formal o informal, quedando por fuera de las titulaciones académicas. De esta manera, y entendiendo que la producción de contenidos en la web se inscribe dentro de una gama de nuevas actividades y profesiones que surgen con el desarrollo de las TD e internet, nos interesó entender cómo era su recorrido formativo para llegar a ser "youtubers";

- creatividad: aquí pusimos el foco en los discursos que circulan sobre este tipo de actividades y que muchas veces encubren relaciones precarias, dando lugar a lo que los estudios críticos refieren como relaciones de explotación. Si bien, esto no resultaba novedoso en sí mismo, si lo era a la luz de entender el alcance de este tipo de actividades y su consolidación en los últimos diez años;

- valores: en línea con la dimensión anterior, este eje indagó en aspectos de la construcción subjetiva de los actores, atendiendo a la literatura que señala una preponderancia de lo dionisíaco (el deseo), por sobre lo apolíneo (la mesura, el equilibrio) en esta etapa del capitalismo. Aquí nos interesaba entender la proyección profesional a largo plazo o no de este tipo de actividades, y el reconocimiento efectivo y esperado;

- vínculos: esta última dimensión puso el foco en las relaciones que se establecían o no dentro de la plataforma de videos. Para esto partimos de la idea de redes de contactos (y reconocimiento, en un espacio de lucha constante por la atención) que el desarrollo de las TD e internet habilita. Esto para entender la dinámica de las relaciones en este tipo de actividades e indagar en posibles acciones para la resolución de conflictos.

Esta propuesta analítica nos sirvió para establecer un recorte frente al mar de información que circula en YouTube y abordar de manera sistemática nuestro objeto de estudio. Aun así, dicha operacionalización resultó algo extensa, lo que generó inconvenientes vinculados sobre todo al último eje de nuestra matriz, la recolección de datos.

El segundo eje propuesto en la matriz consiste en cómo acceder a los sujetos y/o población objetivo. En el caso de la investigación A dicha dificultad radicó en que las instituciones educativas que se encontraban dentro de nuestro listado, aceptaran formar parte de la muestra. Cabe mencionar que en un primer momento consideramos que íbamos a contar rápidamente con el aval de dichas instituciones para ingresar allí. Sin embargo, después de varios intentos fallidos, por ejemplo, no lográbamos establecer una fecha concreta de visita, o la falta de respuesta de las autoridades a nuestros llamados, entre otros. Consideramos necesario diseñar un protocolo acerca de cómo acceder a las instituciones y darles un seguimiento. Dicho protocolo, por decirlo de alguna manera, estaba compuesto por una serie de llamados telefónicos que nos permitía confirmar si en esa institución iba a ser posible realizar el trabajo de campo pautado (contando así con netbooks disponibles y 
una/a profesor/a de informática que nos asistiese). Así, fue necesario, además del llamado, establecer un contacto presencial, por más que la encuesta podía cumplimentarse a través de computadoras o dispositivos digitales, dado que se encontraba online.

Algo de esta dificultad en la obtención de los contactos se replicó en la investigación B. Allí el contacto se establecía directamente con los y las estudiantes a partir de los datos que cada una de las instituciones nos facilitó. Léase correos electrónicos. Entonces, al identificar en el primer envío la baja tasa de respuesta, tuvimos que solicitar nuevamente el armado de los listados con más datos de contacto de los y las ingresantes. Una vez con dichos listados, sumamos entonces el llamado telefónico como estrategia de acceso e incluso el día anterior reconfirmabámos la participación con una nueva comunicación. Así, cabe enfatizar la necesidad e importancia de ponderar en cada estudio las características de los diferentes actores a los que nos interesa acceder.

En el caso de YouTube, la problemática en cuanto al acceso difiere. Como mencionamos brevemente arriba, desconocíamos qué cantidad de productores y seguidores había en el país. Esto volvía difícil, sino imposible, intentar estimar qué cantidad de encuestados/entrevistados debíamos alcanzar para que la muestra sea representativa. Por este motivo, se recurrió al RDS para intentar calcular una pseudo-población que se acercara a la real. Sin embargo, esto tampoco fue efectivo, dado que no contábamos con incentivos que pudieran alentar a nuestros actores a completar la encuesta e invitar a otros a que lo hagan. Es por eso, que se recurrió a otras fuentes para conseguir contactos de productores (a través de sus páginas personales, canales, sitios de redes sociales, etc.), y se viró hacia un abordaje más centrado en lo cualitativo, dado las dificultades en el acceso y la baja tasa de respuesta. Aquí también incidió el hecho de que la mejor manera de enviar los cuestionarios era por mail. Tal como señalamos con la investigación B, este medio, si bien es efectivo para llegar a ciertos sectores etarios, no suele serlo para los más jóvenes.

Este tipo de inconvenientes da cuenta de que según el tipo de unidad de observación con la que estemos trabajando, debemos considerar sus usos y prácticas relativos a las TD e internet. No porque estos constituyan necesariamente nuestro objeto de estudio, sino porque su desatención puede resultar un obstáculo para recolectar la información que necesitamos. En otras palabras, si bien en ocasiones la disponibilidad que permite internet puede facilitar el acceso, esto no implica necesariamente que resulte, tal como vimos en los diferentes ejemplos, ser la mejor solución.

Este eje, lejos de hallarse estabilizado, dialoga también con la velocidad con la que cambian las TD y los usos diversos y novedosos que emergen de tanto en tanto. Claro que algunas de estas dificultades podrían saldarse accediendo a través de sitios de redes sociales. Sin embargo, esto también puede resultar problemático. Por un lado, el hecho de que busquemos generar un contacto a través de los sitios más utilizados no implica necesariamente que sean leídos o contestados. Este tipo de sitios están diseñados en general para interacciones rápidas, y muchas veces los mensajes de gente desconocida (con las cuales no se ha establecido un contacto previo, no forman parte de sus seguidores o no son seguidos 
por ellos/ellas) cae en un espacio poco o nada explorado. Por otro lado, abre una serie de interrogantes vinculados hasta dónde es posible avanzar para acceder a los sujetos y/o población objetivo. Esto lo desarrollamos al final del apartado cuando retomamos las preguntas sobre los aspectos éticos.

El último de los ejes propuestos gira en torno a cómo recolectar datos acerca de prácticas que están mediadas por TD e internet y que pueden acontecer tanto en el ámbito en el privado (relativo a los usos y prácticas asociados a distintos dispositivos), como en el público (plataformas, redes sociales, etc.). En relación a aquellos fenómenos que suceden en el ámbito privado, como las investigaciones A y B, diseñamos una serie de instrumentos de recolección de datos que tenían como propósito reponer parte del mundo analógico y digital de manera separada, pero también combinada. En este sentido, nos preguntamos acerca de las posibles implicancias de utilizar instrumentos de recolección de datos analógicos que buscan captar prácticas asociadas a las tecnologías digitales. Este es el caso, por ejemplo, de los focus groups. Tanto en la investigación A como B, se realizaron distintos focus con estudiantes para entender, en el caso de la primera, los usos y prácticas (entre otras dimensiones) vinculadas a las TD en general y a las netbooks del PCI en particular. En el caso de la segunda, para identificar la operatividad de los saberes tecnosociales que portan dichos actores educativos. Ambos guiones de focus implicaron generar situaciones que permitieran no solo explorar aspectos complejos de expresar verbalmente, sino también que están naturalizados. Así, un punto a mencionar reside en la necesidad de desarrollar herramientas que nos permitan comprender lo gestual y kinestésico, sobre todo en función de captar prácticas asociadas a las TD que se encuentran naturalizadas y que pueden resultar ajenas a quienes investigamos.

Asimismo, durante la investigación A llevamos a cabo una encuesta. Como mencionamos en la presentación del caso, esta consistió en un cuestionario online, administrado a través de LimeSurvey, e implementado a partir de una red interna (intranet $)^{10}$ en cada escuela de manera presencial. Esto implicó que una dupla de investigadoras/es viaje a distintas localidades a lo largo del país. La combinación de estos elementos fue clave para poder alcanzar el número de encuestas esperado. De haberla llevado a cabo de manera remota, seguramente no habríamos podido asegurar el mismo resultado. El motivo de esto reside en algunos de los elementos ya desplegados, pero principalmente, en las dificultades para que, en este caso, jóvenes de entre 15 y 18 años accedieran a completar una encuesta online, la cual, además, al incluir distintas dimensiones, pecaba de ser algo extensa. Nuevamente, las características de los actores, sus posibilidades de acceso (¿todos contaban con internet en las escuelas o en sus casas?) y lo usos que hacen de las TD son elementos que debemos considerar al momento de conducir este tipo de investigaciones.

En la investigación $C$, surgieron otro tipo de inconvenientes vinculados a la recolección de datos. Como relatamos más arriba se confeccionó un cuestionario con el

\footnotetext{
${ }^{10}$ La gran mayoría de las escuelas a las que asistimos, sino todas, no contaban con una conexión estable a internet. En muchos casos, ni siquiera contaban con el servicio.
} 
objetivo de abordar la actividad de los productores de contenidos a partir de distintas dimensiones. Esto generó que en un principio la extensión fuera un obstáculo para las características del tipo de población al que estaba dirigida (mayormente jóvenes con un uso profuso de las TD) e imprimiera sesgos al limitar las posibles respuestas. Dicha limitación implicó, por un lado, recortar el cuestionario y, por otro, recolectar y reconstruir la información ahora faltante de fuentes secundarias. Dada la novedad del fenómeno, la reconfiguración de las preguntas iniciales no resultó ser tan fácil.

Para finalizar, tal como se aprecia en la tabla 1, consideramos que los diferentes ejes dialogan con otro aspecto transversal: la ética. Es decir, ¿hasta dónde podemos acceder?, ¿qué datos son válidos de ser relevados y cuáles no?, ¿dónde está el límite?, ¿cómo podemos establecer aquello que forma parte del ámbito público y aquello que ingresa en el espacio de lo privado dada las características de las TD e internet?

Cuando mencionamos este tema vinculado a la extracción de grandes volúmenes de datos (big data), el conflicto ético respecto del acceso a datos de millones de personas, parece ser más que evidente. Sin embargo, en otro tipo de investigaciones en donde nos limitamos a observar sitios, perfiles, canales de productores, interacciones en plataformas educativas, etc., no reparamos en la cada vez más naturalizada "biovigilancia". La mayoría de las veces, quienes son observados no lo saben. En este sentido, nos preguntamos en qué se distancian este tipo de prácticas. ¿Es solo una cuestión de cantidad, de volumen de información? En cualquier caso, y no solo para pensar la aplicación de la minería de datos en el campo de las ciencias sociales, sino también para revisar nuestras prácticas como investigadoras en niveles más micro.

Otra cuestión vinculada al dilema ético que hemos experimentado, se refiere a la anonimización de perfiles públicos. En la investigación sobre productores de YouTube, por ejemplo, los perfiles de las personas entrevistadas son públicos, y estos se construyen, en la gran mayoría de los casos, sobre una frontera difusa entre privado/público, aquello que Paula Sibilia (2012) denominó "extimidad". Específicamente, nos enfrentamos con algunos problemas a la hora de anonimizar estos perfiles, sobre todo en los casos en que nos interesa mostrar capturas de las interacciones o situaciones que suceden en sus canales y redes sociales, pero también, porque al constituirse como figuras públicas, muchas veces lo que dicen y muestran aparece publicado en distintos medios. Aquí se nos podría objetar que a este tipo de figuras no les importa ser reconocidas. Sin embargo, a veces nos confían datos sensibles (como aquellos relativos a sus ingresos) con la certeza de que sus comentarios van a ser anonimizados.

El último aspecto consiste en cómo traspasar el perfil público/privado que construyen, e indagar en prácticas y discursos que quedan por fuera de aquello que se ve en la pantalla. Aquí, se advierte el desplazamiento constante entre figura on y offline. De cualquier manera, en este caso en particular no nos referimos a cualquier persona sino a quienes crean un trabajo/profesión en este tipo de plataformas y se exponen constantemente. En efecto, muchas veces nos enfrentamos con dificultades para relevar 
datos e información sobre los usos de la plataforma de videos que escapen a la lógica de la exposición y permitan indagar un poco más allá. Lo que nos devuelve nuevamente al inicio, ¿cuál es el límite de esto y cómo hacerlo tangible?

\section{Reflexiones finales}

En este escrito hemos presentado algunas de las discusiones que se (nos) plantean a nivel metodológico en las ciencias sociales y muy especialmente respecto de los instrumentos de recolección de datos cuando estudiamos fenómenos vinculados a las TD e internet. Dichas discusiones, entendemos, guardan cierta correspondencia y en más de una ocasión se entrelazan en una conversación más amplia como es el de las limitaciones de los constructos teóricos que históricamente nos han permitido analizar aquellos datos que producimos, pero que en el capitalismo informacional también experimentan tensiones y se hallan en el foco de la tormenta. Claro que aquí solo recuperamos las limitaciones y desafíos de nuestras experiencias como investigadoras en fenómenos que acontecen a partir de la masificación de las tecnologías digitales e internet en general y los sitios de redes sociales en particular.

Específicamente, en este recorrido hemos, por un lado, caracterizado a las TD y, por otro, recuperado experiencias de investigación que han tensionado y a la vez invitado a repensar(nos) en nuestro quehacer. Nos referimos puntualmente a distintos ejes que presentan dificultades a la hora de conducir este tipo de investigaciones: recorte del objeto, acceso a los sujetos y población objetivo, recolección de datos; y un aspecto transversal a todos estos, las implicancias éticas de nuestro accionar como investigadoras en este campo. Esto, en diálogo con las características específicas que presentan las TD e internet. No obstante, no solo destacamos las limitaciones y obstáculos con los que hemos lidiado, sino también las soluciones que supimos dar para avanzar con nuestras investigaciones.

Asimismo, nos pareció importante presentar, aunque de manera breve dado el objetivo y alcance de este artículo, algunas innovaciones a nivel metodológico como son las etnografías digitales y el uso del Big Data en las ciencias sociales. Entendemos que sobre todo las primeras, plantean una búsqueda en línea con lo que nos propusimos en este artículo, en función de generar técnicas que den cuenta de los fenómenos del mundo en el capitalismo informacional, sumamente atravesado por el uso de las TD e internet. Aun así, entendemos que quedan varias cuestiones por seguir pensando, muchas de ellas vinculadas a los obstáculos y problemas aquí planteados en torno al recorte del objeto, el acceso a los datos, la sistematización de la información, etc. El uso del Big Data, por el momento, parece estar solo accesible para algunas/os investigadoras/es, o para equipos multidisciplinarios. Aun así, es importante que, como sugiere la bibliografía, su uso venga acompañado de reflexiones teóricas críticas que discutan sobre el tipo de datos que estamos produciendo, de qué manera, y con qué fines.

Asimismo, y en base a la inquietud que mencionamos respecto del desarrollo de un mercado privado de la metodología, resulta relevante preguntarnos qué tipo de herramientas elegimos para conducir nuestras investigaciones online, privativas o libres. En este sentido, 
y retomando también los aspectos éticos, nos parece importante enfatizar el uso de instrumentos libres, sobre todo por la implicancia que esto tiene respecto de la información que recolectamos. En las investigaciones presentadas se utilizó (y se prevé utilizar en el caso de la investigación B) un software libre (LimeSurvey) para cargar y administrar los cuestionarios. En muchas investigaciones de carácter público se utilizan formularios de Google, por ejemplo, que si bien pueden resultar fáciles de usar, plantean preguntas respecto de la privacidad de nuestros informantes/encuestados, el cuidado y almacenamiento de los datos, entre otras cuestiones. ¿En qué medida podemos asegurar que estos datos no son tomados por la empresa como insumo para el desarrollo de su modelo de negocios?

En otro orden de ideas, un tema que abordamos lateralmente y que incide en cómo resolvemos los problemas que surgen en este tipo de investigaciones se vincula con la formación que recibimos como cientistas sociales. Nuevamente, el caso del Big Data vuelve evidente que el hecho de que existan este tipo de técnicas y herramientas no garantiza que podamos usarlas. Ya sea porque no tenemos los conocimientos técnicos, porque sus costos resultan prohibitivos, y/o porque implican repensar y problematizar aspectos que no resultan relevantes en su ámbito de surgimiento, no vinculado a la investigación social. En este sentido, se impone replantear los diseños curriculares de aquellas carreras que tengan alguna posible relación, directa o indirecta, con este tipo de fuentes de información.

De alguna manera, si bien el abordaje de estos fenómenos implica una formación continua en varios aspectos (desde cuestiones técnicas hasta las tendencias y cambios de los sitios, plataformas, programas, etc., que estudiamos, así como las prácticas asociadas a los mismos), se vuelve cada vez más evidente que la colaboración multidisciplinaria, así como la asistencia de técnicos/as especializados, resulta fundamental para conducir este tipo de investigaciones.

Para finalizar, tal como hemos señalado a lo largo de estas páginas, las diferentes transformaciones en la producción de conocimiento social en el capitalismo informacional nos invitan, por un lado, a seguir precisando cuáles son aquellos instrumentos que nos permiten construir nuestros objetos de investigación de manera más acabada y, por otro, en el mismo movimiento, a ensayar posibles marcos teóricos. En efecto, nos encontramos frente a una diversidad de dilemas éticos que, lejos de hallarse clausurados, se encuentran en pleno proceso de "ebullición". Aquí, no solo alentamos dichas discusiones, sino que las consideramos centrales para la construcción de una sociedad más igualitaria en la que la producción de conocimiento social no quede únicamente en manos de unos pocos, sino que sea un bien común.

\section{Bibliografía}

BECERRA, Gaston; ALURRALDE, Juan Pablo López. (2017). Big data y Data mining. Un análisis crítico acerca de su significación para las ciencias psicosociales a partir de un estudio de caso. $\{$ PSOCIAL\}, vol.3. n.2, p.66-85. 
BLONDEAU, Oliver. (1999). Génesis y subversión del capitalismo informacional. En: RODRÍGUEZ, Emanuel; SÁNCHEZ, Raúl (Comp.) Capitalismo cognitivo, propiedad intelectual, $y$ creación colectiva. Madrid: Traficantes de sueños.

BOUTANG, Yann. (1999). Riqueza, propiedad, libertad y renta en el capitalismo cognitivo. En: RODRÍGUEZ, Emanuel; SÁNCHEZ, Raúl (Comp.) Capitalismo cognitivo, propiedad intelectual, y creación colectiva. Madrid: Traficantes de sueño

CHARTRAND, Harry. (2005). The Competitiveness of Nations in a Global Knowledge-Based Economy, PhD thesis en Economía. Saskatoon: University of Saskatchewan

CASTELLS, Manuel. (2008). [1997] La era de la información, tomos I, II y III. México: Siglo XXI.

DI PROSPERO, Carolina; DAZA, Daniel. (2019). "Etnografía (de lo) digital Introducción al dossier. Etnografías Contemporáneas, vol.5, n.9

DOLCEMASCOLO, Agostina; DUGHERA, Lucila. (2019). Emprendedores 2.0: de la creatividad a la precariedad en YouTube. In: Revista Intercambios; vol. IV.

DUGHERA, Lucila; DOLCEMASCOLO Agostina; YANSEN, Guillermina. (2020). Institución escolar y Programa Conectar Igualdad: representaciones de docentes respecto de las normas de uso de las tecnologías digitales. In: Virtualidad, Educación y Ciencia (VEC); Año: 2020 p. 43 - 58.

HECKATHORN, Douglas. (1997). Respondent-driven sampling: a new approach to the study of hidden populations. Social Problems, vol.44, n.2, p.174-199.

LARROSA, Jorge. (2006). Sobre la experiencia. Aloma. Revista de Psicologia i Ciències de l'Educació, vol.19, pp.87-112.

MANTECÓN, Alejandro; JUAN, Montse; CALAFAT, Amador; BECOÑA, Elisardo; ROMÁN, Encarna. (2008). Respondent-Driven Sampling: un nuevo método de muestreo para el estudio de poblaciones visibles y ocultas. Adicciones, vol.20, n2.

MENESES ROCHA, María Elena. (2018). Grandes datos, grandes desafíos para las ciencias sociales. Revista mexicana de sociología, vol. 80. n.2, pp.415-444.

PEIRONE, Fernando; DUGHERA, Lucila; BORDIGNON, Fernando. (2019). Saberes tecnosociales emergentes. Hacia una propuesta de estudio. En FINQUELIEVICH, Susana; FELDMAN, Patrícia; GIROLIMO, Patrícia; ODENA, Belén. (Comp.). El futuro ya no es lo que era. Buenos Aires: Teseo Press/Instituto de Investigación Gino Germani.

RULLANI, Enzo. (1999). El capitalismo cognitivo, ¿un déjà-vu? . En: RODRÍGUEZ, Emanuel; SÁNCHEZ, Raúl (Comp.) Capitalismo cognitivo, propiedad intelectual, y creación colectiva. Madrid: Traficantes de sueños.

RUIZ SILVA, Alexander. (2016). La tinta indeleble: desde el espacio autobiográfico. Escuela y sociedad en el espacio autobiográfico. Bogotá: Editorial Aula de Humanidades

SANTOS, Guillermo; THOMAS, Hernán. (2012). Inoculaciones y procesiones y cuarentenas. Configuraciones sociotécnicas de las viruelas en América Latina: funcionamiento y circulación de saberes entre Europa, África y América en el siglo XVIII. Redes, vol.18, n.34, 113-142. 
SAUTÚ, Ruth. (2011). “Acerca de qué es y no es investigación científica en ciencias sociales”. En: WAINERMAN, Catalina; SAUTU, Ruth. (comp.). La trastienda de la investigación. Nueva edición ampliada. Buenos Aires: Editorial Manantial

SASSEN, Saskia. (2002). Towards a Sociology of Information Technology. Current Sociology, v. 50, n. 3, p. 365-388.

SIBILIA, Paula. (2012). La intimidad como espectáculo. Fondo de cultura económica.

VARIAN, Hal. (1998). Markets for Information Goods, en www.sims.berkeley.edu.

VERCELLI, Ariel Hernán. (2006). Aprender la Libertad: el diseño del entorno educativo y la producción colaborativa de los contenidos básicos comunes. Disponible en www.aprenderlalibertad.org/aprenderlalibertad.pdf

ZUKERFELD, Mariano. (2007). Bienes Informacionales y Capitalismo Cognitivo. Conocimiento, información y acceso en el siglo XXI. En: Razón y Palabra № 53.

ZUKERFELD, Mariano. (2010). Capitalismo y Conocimiento. Materialismo Cognitivo, Propiedad Intelectual y Capitalismo Informacional. [Tesis doctoral no publicada, FLACSO]. http://capitalismoyconocimiento.wordpress.com/trilogia-capitalismo-yconocimiento/about/

sobre las autoras

\section{Lucila Dughera}

Becaria posdoctoral CONICET. Doctora en Ciencias Sociales, Facultad Latinoamericana de Ciencias Sociales. Magíster en Ciencias, Tecnología y Sociedad por la Universidad Nacional de Quilmes.

Fecha de recepción 30/09/2020

Fecha de aceptación 17/12/2020 